\title{
Phase preparation by atom counting of Bose-Einstein condensates in mixed states.
}

\author{
R. Graham*, T. Wong, M. J. Collett, S. M. Tan and D. F. Walls \\ Department of Physics, University of Auckland, Private Bag 92019, \\ Auckland, \\ New Zealand
}

We study the build up of quantum coherence between two Bose-Einstein condensates which are initially in mixed states. We consider in detail the two cases where each condensate is initially in a thermal or a Poisson distribution of atom number. Although initially there is no relative phase between the condensates, a sequence of spatial atom detections produces an interference pattern with arbitrary but fixed relative phase. The visibility of this interference pattern is close to one for the Poisson distribution of two condensates with equal counting rates but it becomes a stochastic variable in the thermal case, where the visibility will vary from run to run around an average visibility of $\pi / 4$. In both cases, the variance of the phase distribution is inversely proportional to the number of atom detections in the regime where this number is large compared to one but small compared with the total number of atoms in the condensates.

PACS numbers: 03.75 Fi, 05.30 -d

\section{INTRODUCTION}

The recent experimental realization of weakly interacting Bose-Einstein condensates [1] 3] has stimulated a large amount of theoretical work on the properties of these condensates. Recently, there has been a great interest in the interference and the establishment of a relative phase [1 11] between Bose-Einstein condensates which start in Fock states or as a mixture of coherent states. In this paper we show by numerical and analytical calculations how a relative phase is established between two independent condensates initially in mixed states. In particular we look at the quantum interference when the condensates begin in thermal or Poisson mixed states. There is a significant difference in the visibility of the interference patterns between these two cases.

\section{QUANTUM PHASE BETWEEN INITIAL MIXTURES}

We consider two Bose-Einstein condensates which are dropped on top of each other. This system was first proposed by Javanainen and Yoo [4]. An example of such a

\footnotetext{
*Permanent address: Universität GH Essen, Fachbereich Physik, D45117 Essen, Germany.
}

system is a hot wire grid placed below the condensates which acts as an atom detector since it removes atoms from the condensates as they fall under the influence of gravity. Javanainen and Yoo showed that a spatial interference pattern would be observed illustrating the presence of a relative phase between the falling condensates. We will show here that this relative phase is also present when the initial states of the condensates are no longer a pure Fock state of known number but are a mixture of number states. This corresponds physically to the situation where we are uncertain of the initial number of atoms in each condensate. We shall take this mixture to have a number distribution $P_{n}$ giving an initial density operator of the form

$$
\rho=\sum_{n_{1}=0}^{\infty} \sum_{n_{2}=0}^{\infty} P_{n_{1}}\left|n_{1}\right\rangle\left\langle n_{1}\left|\otimes P_{n_{2}}\right| n_{2}\right\rangle\left\langle n_{2}\right|,
$$

where $n_{1}$ and $n_{2}$ refer to the first and second condensate respectively. The spatial interference is established since when we detect the atoms from the condensates we are unaware which condensate the atom came from. This interference pattern is built up from the spatial detections of individual atoms. After $m$ detections we observe atoms at positions $\left\{x_{1}, \ldots, x_{m}\right\}$. If we denote the field operator for the detection of an atom at $x$ as $\hat{\psi}(x)$ then the joint probability of $m$ detections at the $\left\{x_{1}, \ldots, x_{m}\right\}$ positions is

$$
\begin{aligned}
p^{(m)}\left(x_{1}, \ldots, x_{m}\right)= & \mathcal{N}^{(m)} \operatorname{Tr}\left\{\rho \hat{\psi}^{\dagger}\left(x_{1}\right) \ldots \hat{\psi}^{\dagger}\left(x_{m}\right)\right. \\
& \left.\times \hat{\psi}\left(x_{m}\right) \ldots \hat{\psi}\left(x_{1}\right)\right\},
\end{aligned}
$$

where the symbol $\operatorname{Tr}$ denotes the trace over the $n_{1}$ and $n_{2}$ number states. The normalization $\mathcal{N}^{(m)}$ is defined by

$$
\begin{aligned}
\mathcal{N}^{(m)}= & {\left[\int d x _ { 1 } \ldots d x _ { m } \operatorname { T r } \left\{\rho \hat{\psi}^{\dagger}\left(x_{1}\right) \ldots \hat{\psi}^{\dagger}\left(x_{m}\right)\right.\right.} \\
& \left.\left.\times \hat{\psi}\left(x_{m}\right) \ldots \hat{\psi}\left(x_{1}\right)\right\}\right]^{-1}
\end{aligned}
$$

which is independent of $x_{1}, \ldots, x_{m}$ but will in general depend on $\rho$. We define the field operator for the two condensate system [4, [10] as

$$
\hat{\psi}(x)=\hat{a}_{1}+\sqrt{\Gamma} \hat{a}_{2} e^{-i \phi(x)},
$$

with

$$
\phi(x)=\left(k_{1}-k_{2}\right) x
$$


where $\hat{a}_{1}$ and $\hat{a}_{2}$ are the atom annihilation operators for the first and second condensate respectively with each condensate possessing momentum $k_{1}$ and $k_{2}$. We define the ratio $\Gamma=\gamma_{2} / \gamma_{1}$ where $\gamma_{1}$ and $\gamma_{2}$ are the detection rates for each of the condensates. We shall assume $\gamma_{1} \geq$ $\gamma_{2}$ without restriction of generality.

Substituting equation (11) into (2), we obtain the following expression for the joint probability.

$$
\begin{aligned}
& p^{(m)}\left(x_{1}, \ldots, x_{m}\right) \\
= & \mathcal{N}^{(m)} \sum_{n_{1}=0}^{\infty} \sum_{n_{2}=0}^{\infty} P_{n_{1}, n_{2}}\left\langle n_{1}, n_{2}\right| \hat{\psi}^{\dagger}\left(x_{1}\right) \ldots \hat{\psi}^{\dagger}\left(x_{m}\right) \\
& \times \hat{\psi}\left(x_{m}\right) \ldots \hat{\psi}\left(x_{1}\right)\left|n_{1}, n_{2}\right\rangle
\end{aligned}
$$

where we have written $P_{n_{1}, n_{2}}=P_{n_{1}} P_{n_{2}}$ for convenience. The expression for the joint probability above shows that it is a weighted sum of probabilities over fixed numbers $n_{1}$ and $n_{2}$ of initial numbers of atoms in each condensate. The weighting is determined by the probability distribution of the number of atoms. For very narrow distributions, corresponding to an initial fixed number of atoms in each condensate, we see that this will simplify down to the expression used previously by Javanainen and Yoo [1]. However for broader distributions, for example a thermal distribution, this sum will affect this probability and hence the spatial interference.

\section{A. Visibility conditioned on 1 detection}

Before we show how to numerically generate this interference pattern, we look at the build up of interference for the first two detections. Let us start by considering a joint Fock state. After one detection in which an atom is observed at $x_{1}$, the un-normalized state vector for the system is

$$
\begin{aligned}
\hat{\psi}\left(x_{1}\right)\left|n_{1}, n_{2}\right\rangle= & \sqrt{n_{1}}\left|n_{1}-1, n_{2}\right\rangle \\
& +\sqrt{\Gamma n_{2}} e^{-i \phi\left(x_{1}\right)}\left|n_{1}, n_{2}-1\right\rangle .
\end{aligned}
$$

The joint probability density for detecting atoms at $x$ and $x_{1}$ starting from $\left|n_{1}, n_{2}\right\rangle$ is then proportional to

$$
\begin{aligned}
\left\langle\varphi_{2}(\phi) \mid \varphi_{2}(\phi)\right\rangle= & \left\langle n_{1}, n_{2}\left|\hat{\psi}^{\dagger}\left(x_{1}\right) \hat{\psi}^{\dagger}(x) \hat{\psi}(x) \hat{\psi}\left(x_{1}\right)\right| n_{1}, n_{2}\right\rangle \\
= & n_{1}\left(n_{1}-1\right)+\Gamma^{2} n_{2}\left(n_{2}-1\right) \\
& +2 \Gamma n_{1} n_{2}\left\{1+\cos \left[\phi(x)-\phi\left(x_{1}\right)\right]\right\} . \quad(8
\end{aligned}
$$

Since the initial state of interest is a mixture of Fock states with weights $P_{n_{1}, n_{2}}$, the joint probability for this initial state by Eq. (6) is

$$
\begin{aligned}
p^{(2)}\left(x, x_{1}\right)= & \mathcal{N}^{(2)} \sum_{n_{1}, n_{2}} P_{n_{1}, n_{2}}\left\langle n_{1}, n_{2}\right| \hat{\psi}^{\dagger}\left(x_{1}\right) \hat{\psi}^{\dagger}(x) \\
& \times \hat{\psi}(x) \hat{\psi}\left(x_{1}\right)\left|n_{1}, n_{2}\right\rangle \\
= & \mathcal{N}^{(2)} \sum_{n_{1}, n_{2}} P_{n_{1}, n_{2}}\left(n_{1}\left(n_{1}-1\right)+\Gamma^{2} n_{2}\left(n_{2}-1\right)\right.
\end{aligned}
$$

$$
\begin{aligned}
& \left.+2 \Gamma n_{1} n_{2}\left\{1+\cos \left[\phi(x)-\phi\left(x_{1}\right)\right]\right\}\right) \\
= & \mathcal{N}^{(2)}\left(\left[\left\langle n_{1}^{2}\right\rangle-\left\langle n_{1}\right\rangle\right]+\Gamma^{2}\left[\left\langle n_{2}^{2}\right\rangle-\left\langle n_{2}\right\rangle\right]\right. \\
& \left.+2 \Gamma\left\langle n_{1}\right\rangle\left\langle n_{2}\right\rangle\left\{1+\cos \left[\phi(x)-\phi\left(x_{1}\right)\right]\right\}\right)
\end{aligned}
$$

where we have used angle brackets to denote averages taken over $P_{n_{1}, n_{2}}$. These factorize as we assume that $P_{n_{1}, n_{2}}=P_{n_{1}} P_{n_{2}}$. The conditional probability $p\left(x \mid x_{1}\right)=$ $p^{(2)}\left(x, x_{1}\right) / p^{(1)}\left(x_{1}\right)$ differs from the above only by a $x$ independent factor and we may write

$$
p\left(x \mid x_{1}\right)=\frac{\mathcal{N}^{(2)}}{\mathcal{N}^{(1)}}\left\{1+\mathcal{V} \cos \left[\phi(x)-\phi\left(x_{1}\right)\right]\right\}
$$

where

$$
\mathcal{V}=\frac{2 \Gamma\left\langle n_{1}\right\rangle\left\langle n_{2}\right\rangle}{\left[\left\langle n_{1}^{2}\right\rangle-\left\langle n_{1}\right\rangle\right]+\Gamma^{2}\left[\left\langle n_{2}^{2}\right\rangle-\left\langle n_{2}\right\rangle\right]+2 \Gamma\left\langle n_{1}\right\rangle\left\langle n_{2}\right\rangle}
$$

may be interpreted as the conditional visibility of the interference pattern.

For a thermal distribution we use the following relationship between the second moment and the mean $\bar{m} \equiv\langle m\rangle$,

$$
\left\langle m^{2}\right\rangle=2 \bar{m}^{2}+\bar{m}
$$

Substituting the above equations into Eq. (11) we obtain an expression for the conditional visibility in terms of the means

$$
\mathcal{V}_{\text {thermal }}\left(\bar{n}_{1}, \bar{n}_{2}\right)=\frac{\Gamma \bar{n}_{1} \bar{n}_{2}}{\bar{n}_{1}^{2}+\left(\Gamma \bar{n}_{2}\right)^{2}+\Gamma \bar{n}_{1} \bar{n}_{2}}
$$

which gives a maximum visibility for equal net detection rate in each condensate, i.e. giving a maximum value of one third for $\bar{n}_{1}=\Gamma \bar{n}_{2}$.

Alternatively, for the case of a Poissonian number distribution we use the relationship

$$
\left\langle m^{2}\right\rangle=\bar{m}^{2}+\bar{m}
$$

Proceeding in the same manner as in the thermal case we obtain

$$
\mathcal{V}_{\text {Poisson }}\left(\bar{n}_{1}, \bar{n}_{2}\right)=\frac{2 \Gamma \bar{n}_{1} \bar{n}_{2}}{\left(\bar{n}_{1}+\Gamma \bar{n}_{2}\right)^{2}}
$$

which has a maximum value of one half for $\bar{n}_{1}=\Gamma \bar{n}_{2}$. The values of one third and one half that we have obtained have also been seen in optical experiments where intensity correlations were measured for Poissonian and thermal light sources by Rarity et. al. 12

Let us also consider the limiting case of fixed initial number, where we know that there are exactly $n_{1}$ atoms in one condensate and $n_{2}$ in the other. Then using Eq. (11) the conditional visibility is

$$
\mathcal{V}_{\text {Fock }}\left(n_{1}, n_{2}\right)=\frac{2 \Gamma n_{1} n_{2}}{\left(n_{1}+\Gamma n_{2}\right)^{2}-\left(n_{1}+\Gamma^{2} n_{2}\right)} .
$$


The maximum occurs again when $n_{1}=\Gamma n_{2}$ where the net detection rates for both condensates are equal. This is not surprising since the size of the interference term depends on our lack of knowledge of which condensate a given detected atom comes from. If the net detection rate of one condensate is larger than the other, then we know that the detected atom is more likely to have originated from this particular condensate. Unlike the previous two cases, the maximum visibility depends on $N$ the initial total number of atoms in the condensates. The maximum visibility for the special case of equal number and detection rate for each condensate we have

$$
\left[\mathcal{V}_{\text {Fock }}\right]_{\gamma_{1}=\gamma_{2}, n_{1}=n_{2}}=\frac{1}{2(1-1 / N)} \text {. }
$$

In the limit of large $N$ the conditional visibility approaches that of the Poissonian mixture which has the value of one half. For practical purposes where the number of atoms in each condensate is well over one thousand, the cases of a Poissonian mixture and of initial Fock states are indistinguishable. We plot these conditional visibilities for the different initial conditions as a function of the ratio of initial net detection rates, $\Gamma \bar{n}_{1} / \bar{n}_{2}$ between the two condensates in Fig. (1). To see how the maximum visibility changes as we alter the width of these initial distributions, let us also consider some arbitrary Gaussian distribution with a variance $\sigma^{2}$ and mean $\bar{n}$. The conditional visibility when both condensates start with this Gaussian distribution and equal detection rates is

$$
\mathcal{V}_{\text {Gaussian }}\left(\bar{n}, \sigma^{2}\right)=\frac{\bar{n}^{2}}{\sigma^{2}+2 \bar{n}^{2}-\bar{n}}
$$

This is approximately equal to $\left(2+\sigma^{2} / \bar{n}^{2}\right)^{-1}$ for large $\bar{n}$. For wide distributions $\sigma^{2} \gg \bar{n}^{2}$, we see that the conditional visibility tends to zero. Conversely for narrow distributions where $\sigma^{2} \ll \bar{n}^{2}$, the visibility becomes approximately one half for large $\bar{n}$. In the special case of $\sigma^{2}=\bar{n}$ where we approximate the Poisson distribution by a Gaussian, Eq. (18) yields a visibility of one half which is consistent with the value obtained from the expression for the Poissonian visibility given by Eq. (15).

\section{B. Conditional probability density after $m$ detections}

The conditional probability density $p\left(x \mid x_{1}\right)$ displayed by Eq. (10) can be generalized to an expression governing the probability density of $x$ given the previous $m$ measurements $\left\{x_{1} \ldots x_{m}\right\}$. We can write the operator representing the cumulative effect of $m$ detections as

$$
\begin{aligned}
\hat{\Psi}\left(x_{m}\right) \hat{\Psi}\left(x_{m-1}\right) \ldots \hat{\Psi}\left(x_{1}\right) & =\prod_{j=1}^{m}\left(\hat{a}_{1}+\sqrt{\Gamma} \hat{a}_{2} e^{-i \phi_{j}}\right) \\
& =\sum_{k=0}^{m} \pi_{k}^{(m)}\left(\phi_{1}, \ldots, \phi_{m}\right)
\end{aligned}
$$

$$
\times \hat{a}_{1}^{m-k} \hat{a}_{2}^{k} \Gamma^{k / 2}
$$

where we define $\phi_{k} \equiv \phi\left(x_{k}\right)$ for notational convenience and the coefficients $\pi_{k}^{(m)}\left(\phi_{1}, \ldots, \phi_{m}\right)$ can be found by computing the power series expansion

$$
\prod_{j=1}^{m}\left(1+z e^{-i \phi_{j}}\right)=\sum_{k=0}^{m} \pi_{k}^{(m)}\left(\phi_{1}, \ldots, \phi_{m}\right) z^{k} .
$$

They satisfy the recursion relation

$$
\pi_{k}^{(m+1)}=\pi_{k}^{(m)}\left(1-\delta_{k, m+1}\right)+\pi_{k-1}^{(m)}\left(1-\delta_{k, 0}\right) e^{-i \phi_{m+1}}
$$

where

we have used the notation $\pi_{k}^{(m)} \equiv \pi_{k}^{(m)}\left(\phi_{1}, \ldots, \phi_{m}\right)$ for brevity. In a numerical simulation, the product (21) can be updated after every atomic detection by carrying out polynomial multiplication. The un-normalized state vector after applying the above operator to the initial state $\left|n_{1}, n_{2}\right\rangle$ is

$$
\begin{aligned}
\left|\varphi_{m}\right\rangle= & \sum_{k=0}^{m} \sqrt{\frac{n_{1} ! n_{2} !}{\left(n_{1}-m+k\right) !\left(n_{2}-k\right) !}} \\
& \times \pi_{k}^{(m)} \Gamma^{k / 2}\left|n_{1}-m+k, n_{2}-k\right\rangle .
\end{aligned}
$$

Let us now consider the un-normalized wave-function after the $(m+1)$ 'th detection

$$
\left|\varphi_{m+1}(\phi)\right\rangle=\left(\hat{a}_{1}+\sqrt{\Gamma} \hat{a}_{2} e^{-i \phi}\right)\left|\varphi_{m}\right\rangle
$$

where we have explicitly shown the $\phi$ dependence. The joint probability of $m+1$ detections at the $\left\{x_{1}, \ldots, x_{m}, x\right\}$ positions is

$$
\begin{aligned}
& p^{(m+1)}\left(x_{1}, \ldots, x_{m}, x\right) \\
= & \mathcal{N}^{(m+1)} \operatorname{Tr}\left\{\rho \hat{\psi}^{\dagger}\left(x_{1}\right) \ldots \hat{\psi}^{\dagger}\left(x_{m}\right) \hat{\psi}^{\dagger}(x)\right. \\
& \left.\times \hat{\psi}(x) \hat{\psi}\left(x_{m}\right) \ldots \hat{\psi}\left(x_{1}\right)\right\} \\
= & \mathcal{N}^{(m+1)} \sum_{n_{1}=0}^{\infty} \sum_{n_{2}=0}^{\infty} P_{n_{1}, n_{2}}\left\langle\varphi_{m+1}(\phi) \mid \varphi_{m+1}(\phi)\right\rangle,
\end{aligned}
$$

where

$$
\begin{aligned}
& \left\langle\varphi_{m+1}(\phi) \mid \varphi_{m+1}(\phi)\right\rangle \\
= & \frac{n_{1} !}{\left(n_{1}-m-1\right) !}+\sum_{k=0}^{m-1} \frac{\Gamma^{k+1} n_{1} ! n_{2} !}{\left(n_{1}-m+k\right) !\left(n_{2}-k-1\right) !} \\
& \times\left|\pi_{k}^{(m)} e^{-i \phi}+\pi_{k+1}^{(m)}\right|^{2}+\frac{\Gamma^{m+1} n_{2} !}{\left(n_{2}-m-1\right) !}
\end{aligned}
$$

The conditional probability density is thus 


$$
\begin{aligned}
& p\left(x \mid x_{1}, \ldots, x_{m}\right) \\
= & N^{(m)}\left[\left\langle n_{1}\left(n_{1}-1\right) \ldots\left(n_{1}-m\right)\right\rangle\right. \\
& +\sum_{k=0}^{m-1}\left\langle n_{1}\left(n_{1}-1\right) \ldots\left(n_{1}-m+k+1\right)\right\rangle \\
& \times\left\langle n_{2}\left(n_{2}-1\right) \ldots\left(n_{2}-k\right)\right\rangle \Gamma^{k+1}\left|\pi_{k}^{(m)} e^{-i \phi}+\pi_{k+1}^{(m)}\right|^{2} \\
& \left.+\Gamma^{m+1}\left\langle n_{2}\left(n_{2}-1\right) \ldots\left(n_{2}-m\right)\right\rangle\right]
\end{aligned}
$$

where

$$
N^{(m)}=\frac{\mathcal{N}^{(m+1)}}{p^{(m)}\left(x_{1}, \ldots, x_{m}\right)}
$$

is a $x$ independent normalization factor. The angle brackets denotes the sum over the probability distribution $P_{n_{1}, n_{2}}$.

For different initial mixtures, either a thermal or a Poissonian distribution, we obtain different relationships between the higher order moments and the first (i.e. the mean). The relationship for the thermal case is

$$
\langle n(n-1) \ldots(n-k)\rangle_{\text {thermal }}=(k+1) ! \bar{n}^{k+1}
$$

and

$$
\langle n(n-1) \ldots(n-k)\rangle_{\text {Poisson }}=\bar{n}^{k+1}
$$

for the Poissonian distribution. Using these properties we obtain the conditional probability density for the thermal distribution

$$
\begin{aligned}
p_{\text {Poisson }}\left(x \mid x_{1}, \ldots, x_{m}\right)= & N_{\text {Poisson }}^{(m)}\left[\bar{n}_{1}^{m+1}+\left(\Gamma \bar{n}_{2}\right)^{m+1}\right. \\
& +\sum_{k=0}^{m-1} \mathcal{A}_{k}^{(\text {Poisson })} \\
& \left.\times\left|\pi_{k}^{(m)} e^{-i \phi}+\pi_{k+1}^{(m)}\right|^{2}\right]
\end{aligned}
$$

and

$$
\begin{aligned}
p_{\text {thermal }}\left(x \mid x_{1}, \ldots, x_{m}\right)= & N_{\text {thermal }}^{(m)}\left\{( m + 1 ) ! \left[\bar{n}_{1}^{m+1}\right.\right. \\
& \left.+\left(\Gamma \bar{n}_{2}\right)^{m+1}\right]+\sum_{k=0}^{m-1} \mathcal{A}_{k}^{\text {(thermal })} \\
& \left.\times\left|\pi_{k}^{(m)} e^{-i \phi}+\pi_{k+1}^{(m)}\right|^{2}\right\}
\end{aligned}
$$

for the Poissonian distribution. Note that the $x$ independent normalization factors are now calculated with respect to the relevant density operator, i.e. Poisson for $N_{\text {Poisson }}^{(m)}$ and thermal for $N_{\text {thermal }}^{(m)}$. Both the thermal and Poisson distribution's conditional probability densities have a similar form with different weighting factors $\mathcal{A}_{k}$ given by

$$
\mathcal{A}_{k}^{(\text {Poisson })}=\bar{n}_{1}^{m-k} \bar{n}_{2}^{k+1} \Gamma^{k+1}
$$

and

$$
\mathcal{A}_{k}^{(\text {thermal })}=(m-k) !(k+1) ! \mathcal{A}_{k}^{(\text {Poisson })} .
$$

The generalized conditional probability distributions Eq. (32) and Eq. (33) groups all the measurement dependent terms, the $\left\{\phi_{1}, \ldots, \phi_{m}\right\}$ detections, within the $|\ldots|$ brackets. Numerical calculations are readily obtainable since we need only to simulate the expression within the |... brackets. We shall come back to this point and explain in more detail the numerical simulation process when we describe the numerical results in section IID.

\section{Analytical results for a large number of detections}

\section{Poissonian mixtures}

In the case of Poissonian mixtures the expression (32) can be made more explicit and analytical results can be extracted. As shown by Cirac et. al. [7] it is useful to represent the state after $m$ detections in the form of a P-representation

$$
\begin{aligned}
\rho_{m}= & \int \frac{d \psi}{2 \pi} f_{m}(\psi) \int \frac{d \varphi_{1}}{2 \pi}\left|\alpha_{1} e^{i \varphi_{1}}\right\rangle\left\langle\alpha_{1} e^{i \varphi_{1}}\right| \\
& \otimes\left|\alpha_{2} e^{i\left(\varphi_{1}+\psi\right)}\right\rangle\left\langle\alpha_{2} e^{i\left(\varphi_{1}+\psi\right)}\right|
\end{aligned}
$$

with

$$
\alpha_{1}=\sqrt{\bar{n}_{1}}, \alpha_{2}=\sqrt{\bar{n}_{2}} .
$$

In writing Eq. (36) we are assuming that the observation time $t_{m}$ satisfies $\gamma_{i} t_{m} \ll 1$, so that only a negligible fraction of the atoms in the condensates are counted. The initial Poissonian mixture $\rho_{0}$ is also of the form of Eq. (36) with

$$
f_{0}(\psi)=1
$$

The effect of an additional counting event on (36) is to change

$$
f_{m}(\psi) \rightarrow f_{m+1}(\psi)
$$

with

$$
f_{m+1}(\psi)=\frac{1}{N_{m+1}}\left[1+\lambda \cos \left(\phi_{m+1}-\psi\right)\right] f_{m}(\psi)
$$

where

$$
\lambda=\frac{2 \sqrt{\Gamma \bar{n}_{1} \bar{n}_{2}}}{\bar{n}_{1}+\Gamma \bar{n}_{2}}
$$

and $N_{m+1}$ is determined by normalizing $\int \frac{d \psi}{2 \pi} f_{m+1}(\psi)=$ 1 after each counting event. We note that $0<\lambda \leq 1$. In the following we shall assume that $\lambda<1$, as the case $\lambda=1$ requires a separate mathematical treatment. The 
physical meaning of $f_{m}(\psi)$ is clear from Eq. (36): it is the probability distribution of the relative phase between the two condensate modes. The explicit form of $f_{m}(\psi)$ as a function of $\psi$ is easily obtained from Eq. (38) and Eq. (39) as 1

$$
f_{m}(\psi)=\prod_{k=1}^{m}\left[1+\lambda \cos \left(\phi_{k}-\psi\right)\right] \frac{1}{N_{k}} .
$$

The normalized joint probability distribution to observe the phases $\phi_{1}, \ldots, \phi_{m+1}$ is equal to

$$
\int \frac{d \psi}{2 \pi} \prod_{k=1}^{m+1}\left[1+\lambda \cos \left(\phi_{k}-\psi\right)\right]=\prod_{k=1}^{m+1} N_{k}
$$

Therefore the conditional probability 32 determined by the ratio of 2 joint probabilities is equal to $N_{m+1}$ and given by

$p_{\text {Poisson }}\left(x \mid x_{1}, \ldots, x_{m}\right)=\int \frac{d \psi}{2 \pi}[1+\lambda \cos (\phi-\psi)] f_{m}(\psi)$.

where $\phi=\phi(x)$ and $f_{m}(\psi)$ depends on the previous $m$ detections. Now we investigate the behavior of $f_{m}(\psi)$ as a function of $\psi$ for large $m$. We shall assume that it becomes a narrow distribution centered around some $m$-dependent maximum $\psi_{m}$ with a variance $\sigma_{m}^{2}$, i.e. we put

$$
f_{m}(\psi)=\frac{1}{\sqrt{2 \pi} \sigma_{m}} \exp \left[-\frac{\left(\psi-\psi_{m}\right)^{2}}{2 \sigma_{m}^{2}}\right]
$$

We assume here that $\sigma_{m}^{2} \ll 1$, so that the $2 \pi$-periodicity of $f_{m}(\psi)$ is not in noticeable conflict with (44).

We shall now determine the time evolution of $\psi_{m}$ and $\sigma_{m}^{2}$, using Eq. (39), and show that our assumptions are self-consistent in that indeed $\sigma_{m}^{2} \ll 1$ for $m \gg 1$. From Eq. (41) we obtain by taking the logarithm

$$
\ln f_{m}(\psi)=\sum_{k=1}^{m} \ln \left[1+\lambda \cos \left(\phi_{k}-\psi\right)\right]+c
$$

where the constant $c$ depends on the $\phi_{m}$ but not on $\psi$. The maximum $\psi_{m}$ of $\ln f_{m}(\psi)$ must therefore satisfy

$$
\sum_{k=1}^{m} \frac{\lambda \sin \left(\phi_{k}-\psi_{m}\right)}{1+\lambda \cos \left(\phi_{k}-\psi_{m}\right)}=0 .
$$

\footnotetext{
${ }^{1}$ This formula permits us to appreciate why the case $\lambda=1$ is very special: For $\lambda<1$ the distribution (41) is a positive function $f_{m}(\psi)>0$ everywhere. For $\lambda=1$ it has $m 2$ fold degenerate zeros, i.e. $f_{m}(\psi)$ cannot approach a smooth function for $m \rightarrow \infty$.
}

The evolution of the maximum is obtained similarly by taking the logarithm of Eq. (39) and using Eq. (44). We find

$$
-\frac{\left(\psi_{m+1}-\psi_{m}\right)}{\sigma_{m+1}^{2}}+\frac{\lambda \sin \left(\phi_{m+1}-\psi_{m}\right)}{1+\lambda \cos \left(\phi_{m+1}-\psi_{m}\right)}=0 .
$$

It is clear that $\left(\psi_{m+1}-\psi_{m}\right)$ depends on the outcome of the $(m+1)$ 'th measurement. If $f_{m}(\psi)$ is, in fact, a narrow distribution on the scale $2 \pi$, as we have assumed, then the probability distribution to find $\phi_{m+1}$ in that measurement can be estimated as

$$
P_{m+1}\left(\phi_{m+1}\right) \simeq 1+\lambda e^{-\frac{1}{2} \sigma_{m}^{2}} \cos \left(\phi_{m+1}-\psi_{m}\right)
$$

as follows from Eq. (43) and Eq. (44). Based on this we find

$$
\begin{aligned}
\left\langle\frac{\psi_{m+1}-\psi_{m}}{\sigma_{m+1}^{2}}\right\rangle= & 0 \\
\left\langle\frac{\left(\psi_{m+1}-\psi_{m}\right)^{2}}{\sigma_{m+1}^{4}}\right\rangle= & \lambda^{2} \int \frac{d \phi}{2 \pi} \frac{\sin ^{2} \phi}{1+\lambda \cos \phi} \\
& -\frac{\lambda^{3}}{2} \sigma_{m}^{2} \int \frac{d \phi}{2 \pi} \frac{\cos \phi \sin ^{2} \phi}{(1+\lambda \cos \phi)^{2}} \\
= & 1-\sqrt{1-\lambda^{2}} \\
& +\frac{1}{2} \sigma_{m}^{2} \frac{\left(1-\sqrt{1-\lambda^{2}}\right)^{2}}{\sqrt{1-\lambda^{2}}}
\end{aligned}
$$

where we used $\sigma_{m}^{2} \ll 1$ to expand to first order $\exp \left(-\sigma_{m}^{2} / 2\right)=1-\sigma_{m}^{2} / 2$. We conclude that, if $\lambda$ is bounded away from 1 , for small variance of the phase distribution the variance in the jitter of the average phase $\psi_{m}$ in subsequent measurements is of the order of the square of the variance $\sigma_{m}^{2}$ in the phase distribution, i.e. the position of the maximum is very stable and may be considered as fixed in the limit we consider.

Turning to the time evolution of the variance we obtain again using Eq. (44) on the right hand side of Eq. (39), taking the logarithm and the second derivative with respect to $\psi$

$$
\frac{1}{\sigma_{m+1}^{2}}=\frac{1}{\sigma_{m}^{2}}+\frac{\lambda^{2}+\lambda \cos \left(\phi_{m+1}-\psi_{m}\right)}{\left[1+\lambda \cos \left(\phi_{m+1}-\psi_{m}\right)\right]^{2}}
$$

It shows that typically the inverse variance grows according to

$$
\frac{1}{\sigma_{m+1}^{2}}-\frac{1}{\sigma_{m}^{2}}=O(1)
$$

which is the reason why the variance itself indeed becomes small. Averaging as before we obtain for $\lambda$ bounded away from 1

$$
\left\langle\frac{1}{\sigma_{m}^{2}}\right\rangle=\left[\left(1-\sqrt{1-\lambda^{2}}\right)+O\left(\sigma_{m}^{2}\right)\right] m+\text { const. }
$$


which shows more explicitly how the inverse variance grows on the average and becomes large for large $m$.

Finally, we relate the phase distribution $f_{m}(\psi)$ after $m$ measurements to the observed interference pattern. Experimentally, a phase distribution may be extracted by fitting the observed interference pattern, normalized with respect to its constant part, to the expected density Eq. (43) after $m$ counts

$$
\int \frac{d \psi}{2 \pi}[1+\lambda \cos (\phi-\psi)] f_{m}(\psi)=1+\lambda^{\prime} \cos \left(\phi-\psi_{m}\right)
$$

with

$$
\lambda^{\prime}=\lambda e^{-\frac{1}{2} \sigma_{m}^{2}} .
$$

Here we used the Gaussian form of $f_{m}(\psi)$ assumed in Eq. (44). Equations (53), (54) allow us to extract numbers for $\psi_{m}$ and $\sigma_{m}^{2}$. A weak link in the argument leading to Eq. (53) might seem to be the fact that the conditional probability (43) is proportional to the expectation value of the density in the condensates, while what we really need is a normalized measure of the density of the counted atoms. However, all that is really required is that these two quantities should be proportional to each other, which is an assumption implicitly already made when assuming that the counting rate is proportional to the number operator in the condensate.

\section{Thermal mixtures}

If the initial states of the two condensates are thermal mixtures we may adapt the results for the Poissonian mixtures as follows. The thermal initial state can be represented as a mixture of Poissonian states via

$$
\begin{aligned}
\rho_{0}= & \frac{1}{Z_{1} Z_{2}} \sum_{n_{1}, n_{2}} e^{-\beta\left(n_{1}+n_{2}\right)}\left|n_{1}\right\rangle\left\langle n_{1}|\otimes| n_{2}\right\rangle\left\langle n_{2}\right| \\
= & \frac{1}{\mathcal{N}} \int d^{2} \alpha_{1} \int d^{2} \alpha_{2} \exp \left(-\frac{\left|\alpha_{1}\right|^{2}}{\bar{n}_{1}}-\frac{\left|\alpha_{2}\right|^{2}}{\bar{n}_{2}}\right)\left|\alpha_{1}\right\rangle\left\langle\alpha_{1}\right| \\
& \otimes\left|\alpha_{2}\right\rangle\left\langle\alpha_{2}\right| \\
= & \frac{1}{\mathcal{N}^{\prime}} \int_{0}^{\infty} d x_{1} \int_{0}^{\infty} d x_{2} \exp \left(-\frac{x_{1}}{\bar{n}_{1}}-\frac{x_{2}}{\bar{n}_{2}}\right) \\
& \times \int \frac{d \psi}{2 \pi} f_{0}(\psi) \int \frac{d \varphi_{1}}{2 \pi}\left|\sqrt{x_{1}} e^{i \varphi_{1}}\right\rangle\left\langle\sqrt{x_{1}} e^{i \varphi_{1}}\right| \\
& \otimes\left|\sqrt{x_{2}} e^{i\left(\varphi_{1}+\psi\right)}\right\rangle\left\langle\sqrt{x_{2}} e^{i\left(\varphi_{1}+\psi\right)}\right|
\end{aligned}
$$

with the inverse temperature proportional to $\beta$ and normalization factors $\mathcal{N}$ and $\mathcal{N}^{\prime}$. The evolution of this initial state due to the counting of atoms can now be obtained by using the evolution for Poissonian mixtures for fixed Poissonian averages $x_{1}, x_{2}$ under the integrals over $x_{1}, x_{2}$. We obtain with $f_{0}(\psi)=1$, using Eq. (41) for

$$
\begin{gathered}
\lambda=\lambda\left(x_{1}, x_{2}\right)=\frac{2 \sqrt{\Gamma x_{1} x_{2}}}{x_{1}+\Gamma x_{2}} \\
f_{m}(\psi) \sim \prod_{k=1}^{m}\left[x_{1}+\Gamma x_{2}+2 \sqrt{\Gamma x_{1} x_{2}} \cos \left(\phi_{k}-\psi\right)\right] .
\end{gathered}
$$

The optimal visibility $\lambda$ thus varies according to Eq. (56) for different members of the ensemble whose $x_{1}, x_{2}$ values are exponentially distributed according to Eq. (55). The average visibility becomes

$$
\begin{aligned}
\bar{\lambda} & =\frac{2 \sqrt{\Gamma}\left\langle\sqrt{x_{1} x_{2}}\right\rangle}{\left\langle x_{1}\right\rangle+\Gamma\left\langle x_{2}\right\rangle} \\
& =\frac{\pi}{4} \frac{2 \sqrt{\Gamma\left\langle x_{1}\right\rangle\left\langle x_{2}\right\rangle}}{\left\langle x_{1}\right\rangle+\Gamma\left\langle x_{2}\right\rangle}
\end{aligned}
$$

which may be written as?

$$
\bar{\lambda}=\frac{\pi}{4} \lambda .
$$

For equal average counting rates

$$
\bar{n}_{1}=\Gamma \bar{n}_{2}
$$

the average visibility for initially thermal mixtures is simply

$$
\bar{\lambda}=\frac{\pi}{4},
$$

which agrees well with the result of the numerical simulations to be presented below.

\section{Numerical results}

The form of the expressions for the conditional probabilities, Eq. (32) and Eq. (33) of section (IIB) can be readily applied to stochastic simulations of atom detection which generates interference patterns. The procedure follows the spirit of Javanainen and Yoo's work; a random number $\phi_{1}$ is generated for the initial atom detection (since we know the initial conditional probability distribution is uniform), then it is used to calculate the conditional probability density $p\left(\phi \mid \phi_{1}\right)$ so that the second detection $\phi_{2}$ can be generated by randomly selecting a value according to $p\left(\phi \mid \phi_{1}\right)$. This process is repeated to generate $m$ atom detections which are binned and displayed as an interference pattern.

Examples of these stochastically generated interference patterns are displayed in fig. 2 as histograms of the raw output from the simulations, the sequence of detected

\footnotetext{
${ }^{2}$ This is consistent with the visibility defined by Eq. (40) previously since $\lambda\left(\left\langle x_{1}\right\rangle,\left\langle x_{2}\right\rangle\right) \equiv \lambda$.
} 
positions $\left\{\phi_{1}, \ldots, \phi_{m}\right\}$ are sorted into 25 bins plotted as circles. Figure 2 (a) shows the interference pattern for an initial thermal mixture which has a visibility of 0.79 whereas in fig.2(b) we have a visibility of 0.97 for the Poissonian mixture. These visibilities are calculated via a least-squares fit of the form $1+\beta \cos (2 \pi+\phi)$ shown as the solid curves in fig. 2(a) and (b). In both cases we have simulated 500 detections.

The numerical simulations used to generate the histograms in fig. 2(a) and (b) also calculate the conditional probability distributions before each detection as these are necessary to calculate the location of the detected atom. The evolution of these conditional distributions gives an insight into the build up of the interference pattern [10]. The visibility of the complete interference pattern (as calculated from the least-square fit) can be considered as an average over these conditional visibilities. Graphs of the conditional visibility as a function of atom detections typically approach a value close to 1 within 100 detections and stay at that value thereafter. Thus the conditional visibility after $m$ detections can be thought of as a good approximation of the visibility of the complete interference pattern for values of $m>100$. We have not displayed these graphs of stochastically generated sequences since they possess fluctuations about a generic shape which is a property of all such sequences. We plot the average conditional visibility over many such sequences in fig. 3 and fig. 4 for Poisson and thermal mixtures respectively. The ratio $\Gamma \bar{n}_{2} / \bar{n}_{1}$ was set at 1 with the average performed over 1000 runs (we shall hereby refer to an individual sequence of detections as a single "run" for convenience). The shaded regions around the average conditional visibility displayed as a solid line depict the extent of the fluctuations for an individual run. The boundaries of this shaded region corresponds to the upper and lower quartiles respectively, $25 \%$ of the data lies below the lower quartile whereas $25 \%$ lies above the upper quartile, thus the probability that the fluctuations lie within this region is $50 \%$. These fluctuations are much larger for the thermal mixtures in comparison with those of the Poissonian mixtures.

The maximum value of the visibility in fig. 3 occurs at 500 atoms detected with a value of 0.999 (3 sig. fig.). The value of the visibility obtained from Eq. (54) using $\sigma_{m}^{2} \sim 1 / m$ is 0.999 (3 sig. fig.) which agrees very well with the simulation. In the case of the thermal mixture, fig. 战, the maximum value of the visibility is 0.777 again at 500 detections. This is close to the analytical value of $\pi / 4 \approx 0.785$ predicted by Eq. (59).

The large difference in the fluctuations between the two cases is due to their differing degree of sensitivity to particular runs. In the Poisson case, it is relatively insensitive since the majority of the terms in the $|\ldots|$ brackets of Eq. (32) depends on the $\left\{\phi_{1}, \ldots, \phi_{m}\right\}$ detections fairly equally whereas in the thermal case we see there is an additional factorial factor in front of the $|\ldots|$ brackets which favors $k$ values at the ends (close to zero and $m-1)$. This enhances the sensitivity of the visibility upon particular combinations of $\phi_{k}$ thus the sensitivity is high. Alternatively, the large fluctuations of the visibility in the thermal case is not surprising when we consider the analytic treatment of section (IIC/2). The thermal state was represented as a mixture of Poissonian states so that the averaged conditional visibility has an additional average over the mixture of Poissonian states.

The variance for the Poisson case can be estimated from fig. 3 by using Eq. (54) since the fluctuations are small. When the variance are small compared to 1 , the variance is

$$
\sigma_{m}^{2}=2\left(\lambda-\lambda^{\prime}\right)
$$

where $\lambda^{\prime}$ is the numerical averaged visibility and in the case of equal counting rates $\lambda=1$. The variance for the Poisson case is graphed as the solid curve in fig. 5 with the dashed curve displaying the $\sigma_{m}^{2} \sim 1 / m$ relationship predicted by Eq. (52). Note that we have not plotted variances below 50 detections since Eq. (54) is only valid for number of detections $m \gg 1$. As expected, the agreement between the two curves becomes better as more atoms are detected. We cannot obtain a good estimate of the variance in the same manner for the thermal case because the fluctuations in the visibility are large.

We will show in the appendix that the results for measurements induced phase distribution for initial number states are the same as those obtained for the Poissonian mixtures. Numerical simulations of the time evolution and effect of detections on the wave-function of the condensates have been performed [10] where the variance can be calculated directly from this wave-function. This is possible in the simpler case of initial number states. Because the phase is periodic it is convenient to use the following measure of the spread of the phase distribution:

$$
\delta \phi=1-\langle\cos \Delta \phi\rangle^{2}-\langle\sin \Delta \phi\rangle^{2}
$$

where the trigonometric operators are defined as

$$
\cos \Delta \phi=\frac{1}{2}\left(\widehat{e}^{i \Delta \phi}+\widehat{e}^{i \Delta \phi}\right)
$$

and

$$
\sin \Delta \phi=\frac{1}{2 \imath}\left(\widehat{e}^{i \Delta \phi}-\widehat{e}^{i \Delta \phi}\right) .
$$

with the SG phase operators $\widehat{e}^{i \Delta \phi}$ defined for the relative phase between the two condensates. This measure ranges from zero to one with values close to zero agreeing well with the actual variance. Since our expressions are only valid for small variances, this measure is very useful as an estimate of the variance. Figure 6 plots three curves of $\delta \phi$, each one with differing relative count rates between the condensates $\left(\Gamma N_{2} / N_{1}\right)$ where $N_{1}$ and $N_{2}$ are the initial atom number in the first and second condensate respectively. Analytical predictions of the gradient for each relative count rate are obtained from Eq. (52). The solid, dashed and dash-dotted curves of fig. 6 corresponds to $\Gamma N_{2} / N_{1}$ ratios of $1,1 / 2$, and $1 / 4$ which are 
predicted to have gradients $1,2 / 3$, and $2 / 5$ respectively. The numerical gradient was calculated for points from 20 detections onwards, the points below 20 were ignored because the predictions required $m \gg 1$. The curves clearly show larger curvature in this region in comparison to points after 20 . We obtained numerical gradients of $0.97,0.66$, and 0.41 for the $\Gamma N_{2} / N_{1}$ ratios; $1,1 / 2$, and $1 / 4$. This good agreement between the analytic and numerical gradients verifies the relationship of the visibility $\lambda$ with the relative counting rates, Eq. 40,56, A3). Note that if we had graphed $\delta \phi$ itself instead of its inverse, we would had seen a curve shaped like that of fig. 5, hence the straightness of the curves of fig. 6 indicates the accuracy of the $1 / m$ prediction of the previous section (II $)^{3}$.

\section{SUMMARY}

We have analyzed in detail the build up in quantum coherence between two Bose-Einstein condensates which are initially in a thermal or Poisson state. Interference patterns are produced via spatial atom detections which establishes an arbitrary but fixed relative phase between the condensates. In the regime where the total number of atoms detected is only a negligible fraction of the atoms in the condensates although the actual number of detections is much greater than 1, we find that the visibility of the interference pattern for the Poisson distribution depends on the relative counting rates of each condensate with a maximum of one for equal rates. In the thermal case, the visibility becomes a stochastic variable which varies from run to run around an averaged value determined again by the relative counting rates but the average has a maximum of $\pi / 4$ for equal rates in good agreement with our numerical simulations. The inverse variance of the phase distribution grows linearly with the number of detected atoms $m$ in the regime where $1 \ll m \ll N_{1}, N_{2}$. This has been shown analytically for the Poisson state and this relationship also holds true for the thermal state since we may write the thermal state as a mixture of Poisson states. In the appendix we have shown that the results for the initial number states follows those derived for the Poisson state. In particular, the inverse variance is proportional to $m$, which has been numerically verified.

This research was supported by the University of Auckland Research Committee, the New Zealand Lottery Grants Board and the Marsden Fund of the Royal Society of New Zealand.

\footnotetext{
${ }^{3}$ Note that section (II C) consider Poisson and thermal states but fig. 6 show results from simulation of initial number states, however we will show in the appendix that the width of the phase distribution has the same relationship with the number of detections $m$ as the Poisson state in the limit $1 \ll m \ll N_{1}, N_{2}$.
}

\section{APPENDIX A: INITIAL NUMBER STATES}

Let us consider the case of initial number states and compare the results with those of initial Poissonian mixtures. The initial number state

$$
\left|\psi_{0}\right\rangle=\left|N_{1}\right\rangle\left|N_{2}\right\rangle
$$

evolves into

$$
\left|\psi_{m}\right\rangle=\sum_{n=0}^{m} C_{n}(m)\left|N_{1}-n\right\rangle\left|N_{2}-m+n\right\rangle
$$

where $C_{n}(0)=\delta_{n, 0}$. We shall assume $\gamma_{1} N_{1}>\gamma_{2} N_{2}$ and that the parameter

$$
\lambda_{N}=\frac{2 \sqrt{\Gamma N_{1} N_{2}}}{N_{1}+\Gamma N_{2}}
$$

is bounded away from 1 . We shall only consider the case

$$
1 \ll m \ll N_{1}, N_{2} .
$$

Then the evolution of the $C_{n}(m)$ under the atom counting process is

$$
\begin{aligned}
C_{n}(m+1) \simeq & \sqrt{N_{1}}\left(1-\delta_{n, 0}\right) C_{n-1}(m) \\
& +\sqrt{\Gamma N_{2}}\left(1-\delta_{n, m+1}\right) e^{-i \phi_{m+1}} C_{n}(m) .
\end{aligned}
$$

We introduce a phase-representation by the Fourier transform

$$
\begin{aligned}
F_{m}(\varphi) & =\sum_{n=0}^{m} C_{n}(m) e^{-i n \varphi} \\
C_{n}(m) & =\int \frac{d \varphi}{2 \pi} e^{i n \varphi} F_{m}(\varphi) .
\end{aligned}
$$

The number density after $m$ measurements is then

$$
\begin{aligned}
P_{m}(x) \simeq & \left\langle\psi_{m}\left|\hat{\psi}^{\dagger}(x) \hat{\psi}(x)\right| \psi_{m}\right\rangle \\
\simeq & 1+\lambda_{N} \operatorname{Re}\left\{\sum_{n=0}^{m}\left(1-\delta_{n, 0}\right)\right. \\
& \times \int \frac{d \varphi}{2 \pi} \int \frac{d \varphi^{\prime}}{2 \pi} F_{m}^{*}(\varphi) F_{m}\left(\varphi^{\prime}\right) e^{-i n\left(\varphi-\varphi^{\prime}\right)} \\
& \left.\times e^{i[\varphi-\phi(x)]}\right\}
\end{aligned}
$$

which for $m \gg 1$ can be approximated by

$$
P_{m}(x) \simeq 1+\lambda_{N} \int \frac{d \varphi}{2 \pi}\left|F_{m}(\varphi)\right|^{2} \cos [\varphi-\phi(x)] .
$$

This formula should be compared with Eq. (43) for Poissonian mixtures. It can then be seen that $\left|F_{m}(\varphi)\right|^{2}$ and $f_{m}(\phi)$ play identical roles. The evolution of $F_{m}(\varphi)$ under the atom counting process follows from Eq. (A5) and (A6) 


$$
F_{m+1}(\varphi) \simeq\left(\sqrt{N_{1}} e^{-i \varphi}+\sqrt{\Gamma N_{2}} e^{-i \phi_{m+1}}\right) F_{m}(\varphi)
$$

and hence, providing a normalization factor $N_{m+1}$ to keep $\left|F_{m+1}(\varphi)\right|^{2}$ normalized if $\left|F_{m+1}(\varphi)\right|$ is

$$
\left|F_{m+1}(\varphi)\right|^{2} \simeq\left[1+\lambda_{N} \cos \left(\phi_{m+1}-\varphi\right)\right]\left|F_{m}(\varphi)\right|^{2} \frac{1}{N_{m+1}}
$$

which, to the accuracy we have considered here, is the same as Eq. (39) for Poissonian mixtures. Hence, the results for the measurement induced phase distribution for number states in the limit $1 \ll m \ll N_{1}, N_{2}$ are the same as the results for Poissonian mixtures obtained in section IIC.

[1] M. H. Anderson, J. R. Ensher, M. R. Matthews, C. E. Wieman, and E. A. Cornell, Science 269, 198 (1995).

[2] C. C. Bradley, C. A. Sackett, J. J. Tollett, and R. G. Hulet Phys. Rev. lett.75, 1687 (1995).

[3] K. B. Davis, M.-O. Mewes, M. R. Andrews, N. J. van Druten, D.S. Durfee, D.M. Kurn and W. Ketterle, Phys. Rev. Lett. 75, 3969 (1995).

[4] J. Javanainen and S. M. Yoo, Phys. Rev. Lett., 76,161 (1996).

[5] M. Naraschewski, H. Wallis, A. Schenzle, J.I. Cirac and P. Zoller, Phys. Rev. A 54, 2185 (1996).

[6] Y. Castin and J. Dalibard (unpublished).

[7] J. I. Cirac, C. W. Gardiner, M. Naraschewski, and P. Zoller, Phys. Rev. A 54, 3714R (1996).

[8] S. M. Barnett, K. Burnett and J. A. Vaccarro, Journal of Research of the National Institute of Standards and Technology 101, 593 (1996).

[9] M. W. Jack, M. J. Collett and D. F. Walls, Phys. Rev. A 54, 4625R (1996).

[10] T. Wong, M. J. Collett and D. F. Walls, Phys. Rev. A 54, 3718R (1996).

[11] K. Mølmer, Phys. Rev. A (to be published).

[12] J. G. Rarity, P. R. Tapster and R. Loudon, (preprint).

FIG. 1. The conditional visibility curves for an initial number state $(n=20)$, Poisson and thermal states plotted as dashed, solid and dash-dotted curves respectively. The visibility is dependent upon the ratio of the mean counting rates between the condensates $\left(\Gamma \bar{n}_{2} / \bar{n}_{1}\right)$.

FIG. 2. Histogram of 500 numerically generated atomic detections plotted as circles. The solid curve is a least-squares fit of the form $1+\beta \cos (2 \pi x+\phi)$. A thermal initial state is shown in (a) and an initial Poisson state is shown in (b).
FIG. 3. Plot of the conditional visibility averaged over one thousand runs versus the number of atomic detections for an initial Poisson state where $\bar{n}_{1}=\Gamma \bar{n}_{2}$. The shaded region corresponds to the interquartile range of the individual runs with the mean over all runs depicted by the solid line. Therefore $50 \%$ of the run lies within this shaded region.

FIG. 4. Plot of the conditional visibility averaged over one thousand runs versus the number of atomic detections for an initial thermal state where $\bar{n}_{1}=\Gamma \bar{n}_{2}$. The shaded region corresponds to the interquartile range of the individual runs with the mean over all runs depicted by the solid line. Therefore $50 \%$ of the run lies within this shaded region. We also plot a dashed line at $\pi / 4$ corresponding to the value of the average visibility predicted from the analytical work.

FIG. 5. Plot of variance versus the number of detected atoms for the Poisson state shown as the solid line. The dashed line is a plot of $\sigma_{m}^{2} \sim 1 / m$ for comparison.

FIG. 6. Plot of $\delta \phi$ versus number of atomic detections with $\Gamma \bar{n}_{2} / \bar{n}_{1}=1,1 / 2$ and $1 / 4$ for the solid, dashed and dash-dotted curves respectively. 
Figure 1

R. Graham, T. Wong, M. J. Collett, S. M. Tan and D. F. Walls

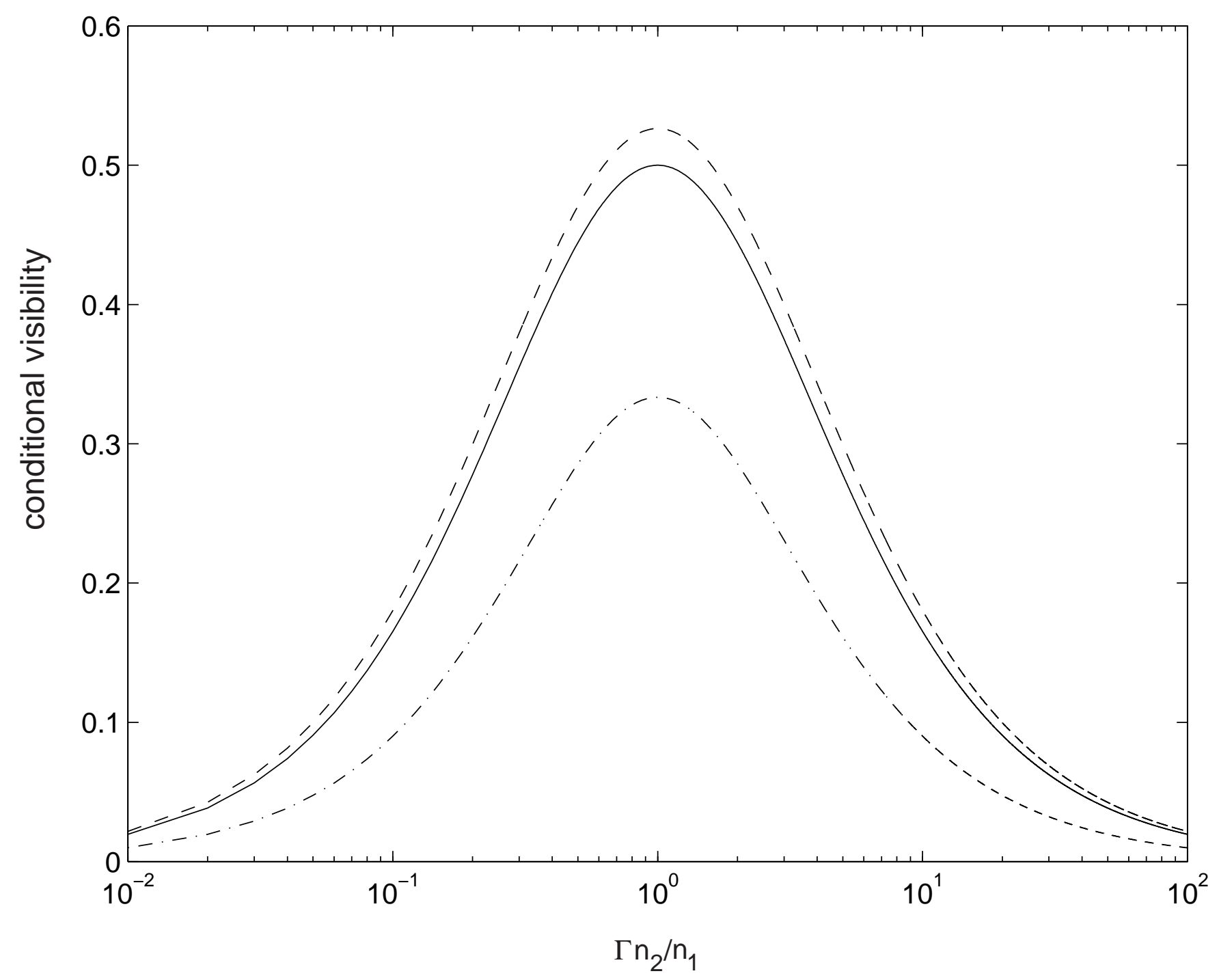


Figure 2

R. Graham, T. Wong, M. J. Collett, S. M. Tan and D. F. Walls
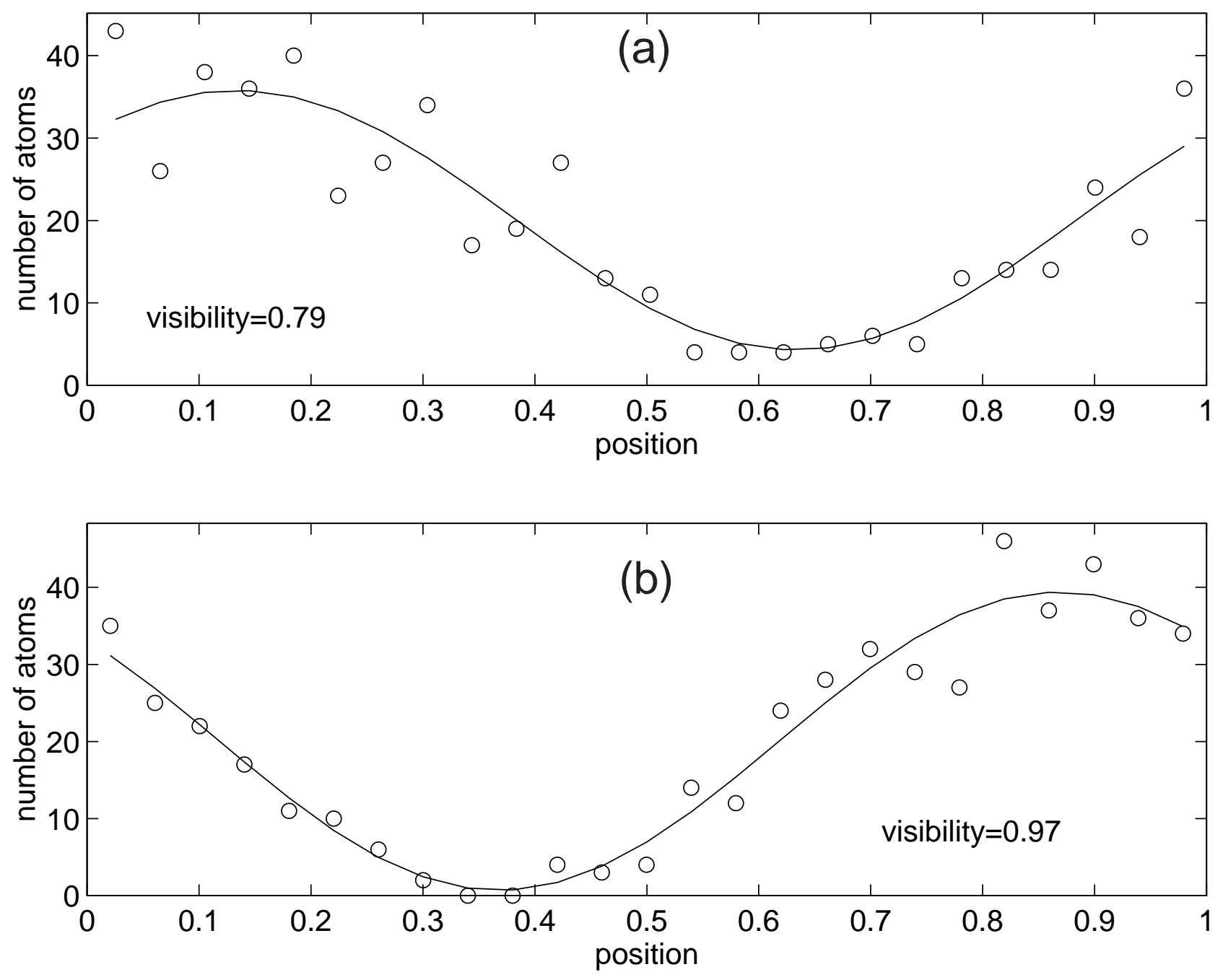
Figure 3

R. Graham, T. Wong, M. J. Collett, S. M. Tan and D. F. Walls

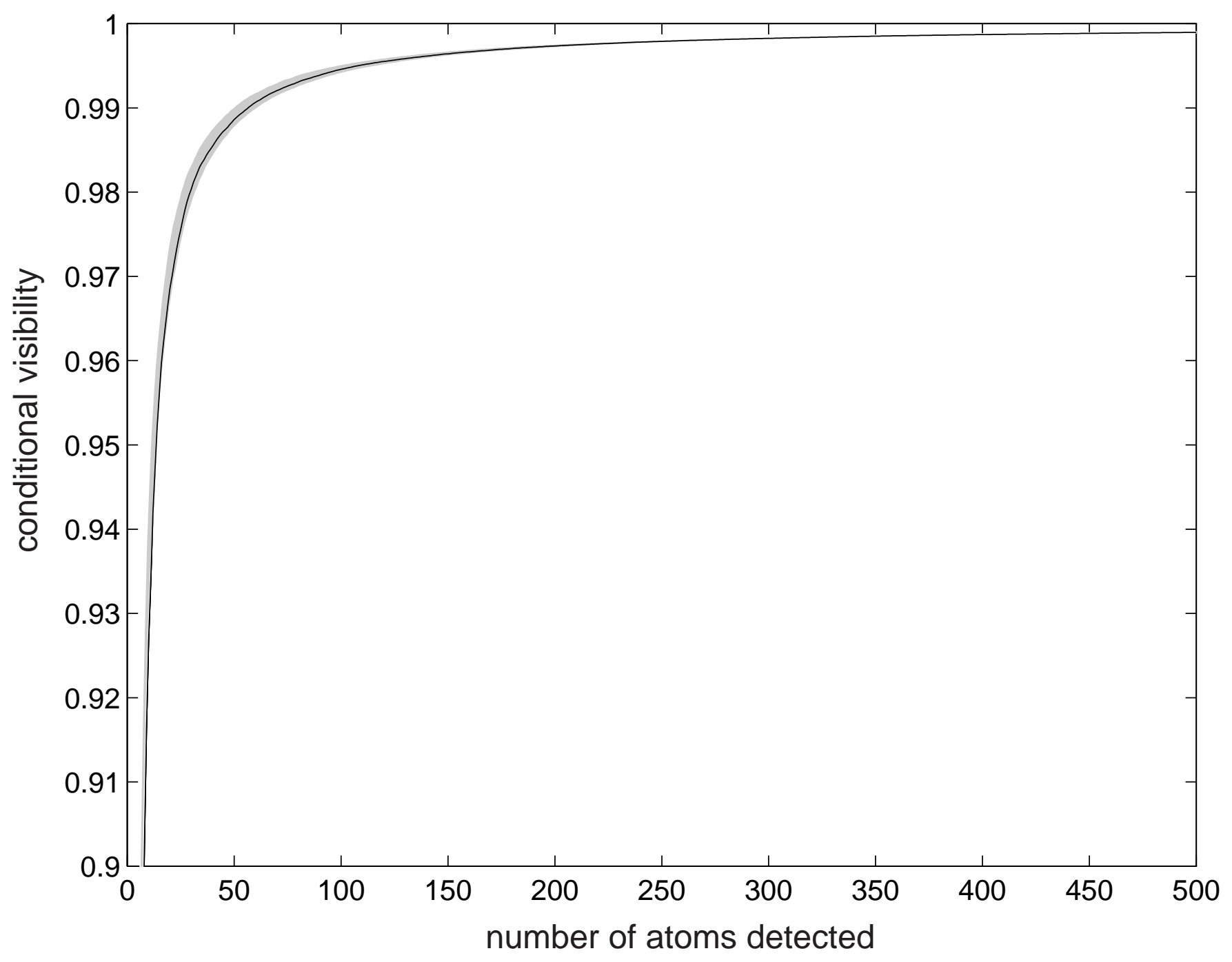


Figure 4

R. Graham, T. Wong, M. J. Collett, S. M. Tan and D. F. Walls

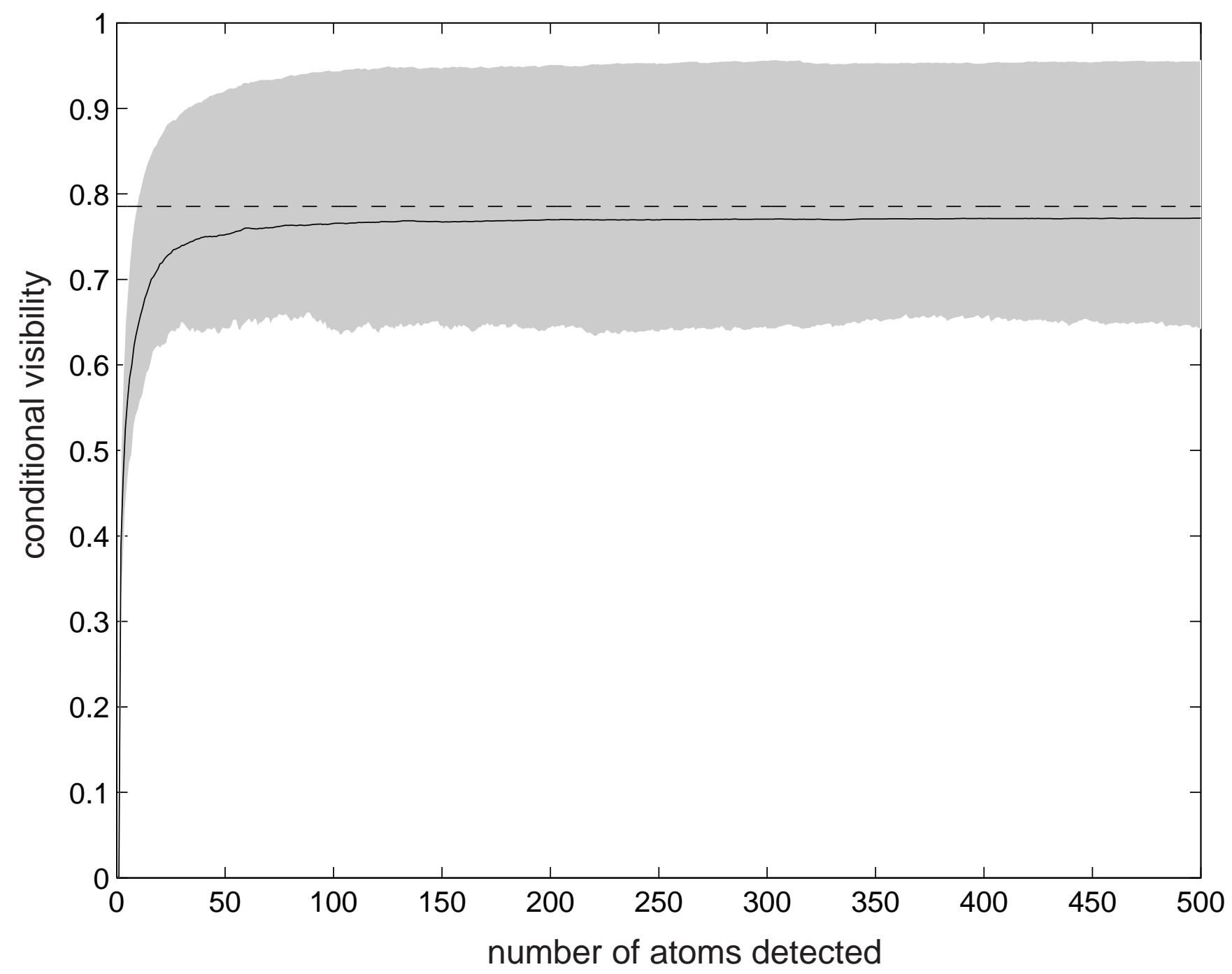


Figure 5

R. Graham, T. Wong, M. J. Collett, S. M. Tan and D. F. Walls

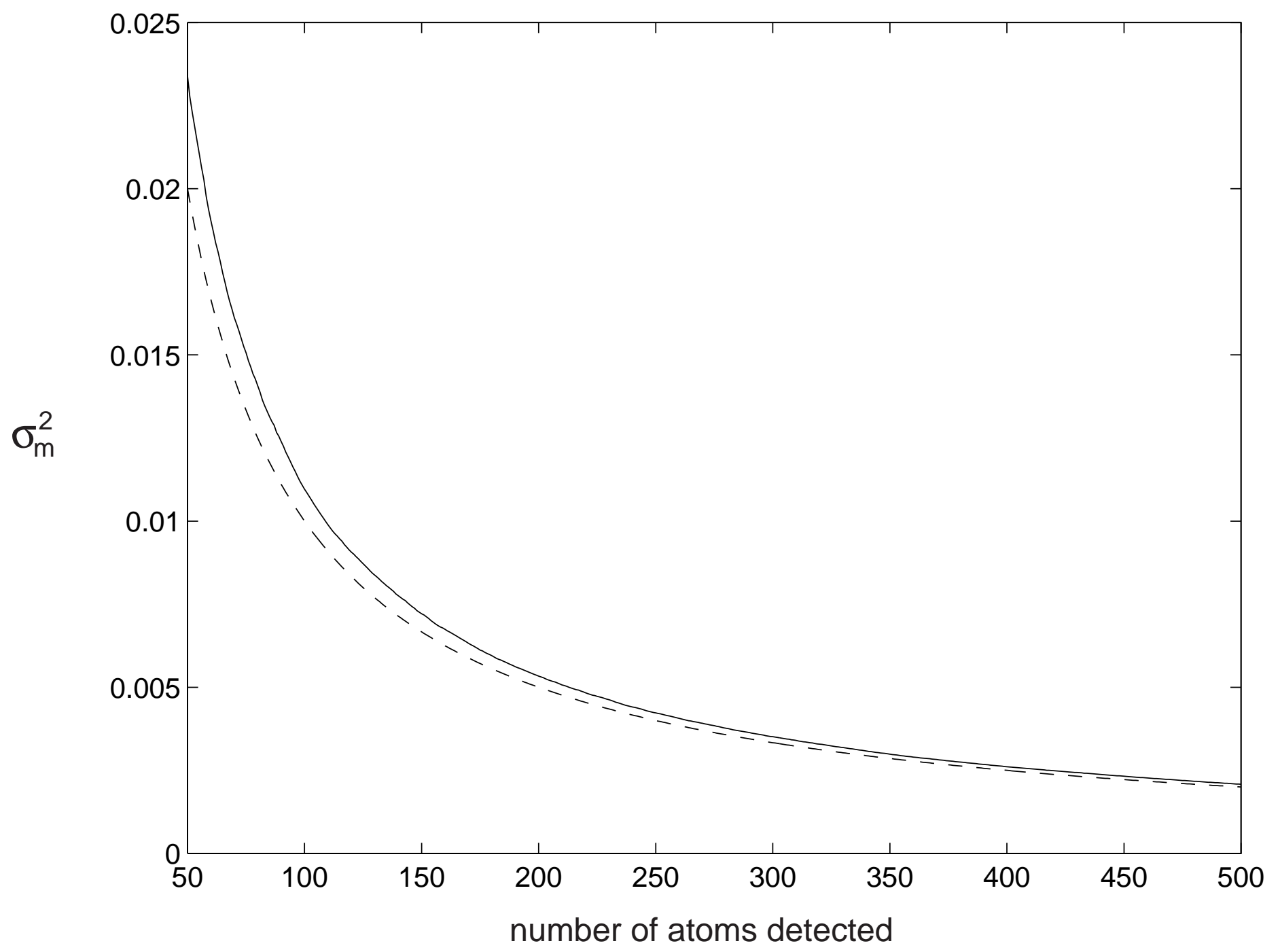


Figure 6

R. Graham, T. Wong, M. J. Collett, S. M. Tan and D. F. Walls

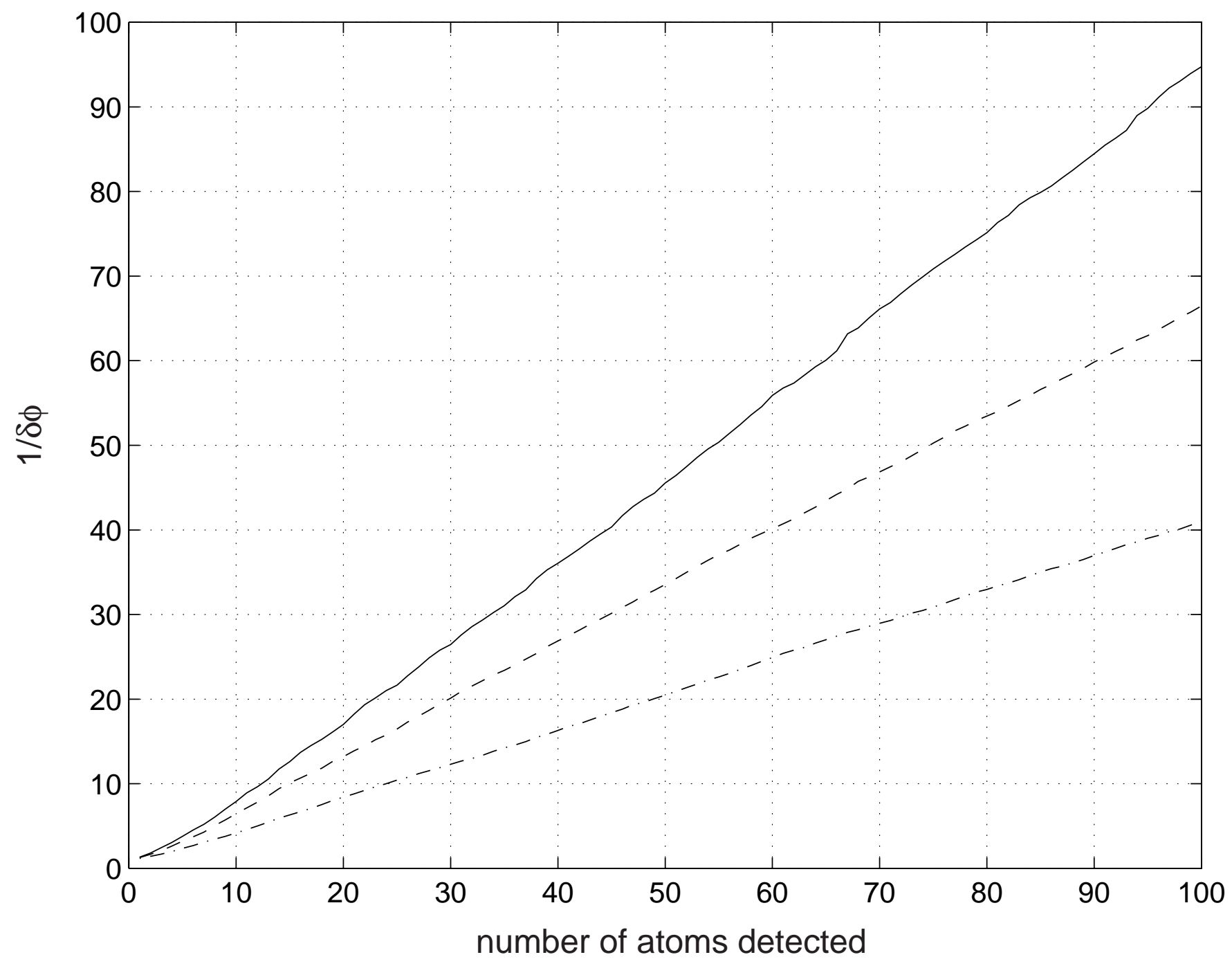

